\title{
Multimodality Imaging of Left Circumflex Artery to Coronary Sinus Fistula
}

\author{
Tan Ling Sze ${ }^{1}$; Yang Faridah Abdul Aziz ${ }^{1}$; Norzailin Abu Bakar ${ }^{1,2,{ }^{*}}$; Fadhli Mohd Sani ${ }^{1}$; Hamid \\ Oemar $^{1,3}$ \\ ${ }^{1}$ Department of Biomedical Imaging, Faculty of Medicine, University of Malaya, Kuala Lumpur, Malaysia \\ ${ }^{2}$ Department of Radiology, University Kebangsaan Malaysia Medical Centre, Kuala Lumpur, Malaysia \\ 3 Department of Cardiology, Faculty of Medicine, University Institute of Technology of Malaysia, Selangor, Malaysia \\ ${ }^{*}$ Corresponding author: Norzailin Abu Bakar, Department of Biomedical Imaging, Faculty of Medicine, University of Malaya, Kuala Lumpur, Malaysia. Tel: +60-123-245709, Fax: +60- \\ 391-737824, E-mail: norzailinabubakar@yahoo.com
}

Received: June 20, 2012; Revised: August 10, 2012; Accepted: August 22, 2012

\begin{abstract}
Coronary artery fistula (CAF) is a rare anomaly of the coronary artery. Patients with this condition are usually asymptomatic. However cardiac failure may occur later in life due to progressive enlargement of the fistula. Diagnosis is traditionally made by echocardiogram and conventional angiogram. However with the advantage of new technologies such as computed tomography (CT) coronary angiography, the course and communications of these fistulae can be delineated non-invasively and with greater accuracy. We report a case of a left circumflex artery fistula to the coronary sinus which was suspected on echocardiogram and the diagnosis was clinched on ECG-gated CT.

Keywords: Circumflex Artery; Coronary Sinus; Fistula; Computed tomography
\end{abstract}

\section{Introduction}

Coronary artery fistula (CAF) is an abnormality in the termination of coronary artery and it was first described in 1865 by Krause (1). It is a communication between one or more coronary arteries and a cardiac chamber or great vessel resulting in a left to right shunt. CAF is rare with an estimated prevalence of $0.002 \%$ in the general population and 0.05-0.25\% among patients who undergo coronary angiography (2). The majority of CAF originate from the right coronary artery and drain into the right ventricle (3). Herein, we report a rare case of CAF from the left circumflex artery to the coronary sinus.

\section{Case Presentation}

A 35-year-old woman with no co-morbidity was referred to the cardiology unit for palpitations that she had been experiencing since childhood. The episode occurred at rest, lasting for less than 10 minutes and happened 2-3 times a week. It was sometimes associated with cyanosis. She denied having any chest pain or symptoms of heart failure.

On examination, her hemodynamic status was stable. There was a soft pan-systolic murmur at the precordial area and grade- 1 continuous murmur at the left sternal border. Transthoracic 2-dimensional echocardiography demonstrated dilation of both the atrium and left circumflex artery with a maximum diameter of $15 \mathrm{~mm}$ Color Doppler echocardiography showed the course of the left circumflex artery emptying into the coronary sinus (Figure $1 \mathrm{~A}$ ). The maximum diameter of the coronary si- nus diameter was $14.4 \mathrm{~mm}$. Continuous turbulence flow on color Doppler at the level of coronary artery-coronary sinus connection as well as in the coronary sinus indicated left-to-right shunt flow (Figure 1 B)

For further delineation of the fistulous communication, ECG-gated contrast-enhanced coronary CT angiography was performed. The patient subsequently underwent an ECG-gated contrast-enhanced coronary CT angiography study using a dual-tube 64-slice multi-detector CT (Siemens, Erlangen Germany). As per standard protocol in our institution, to achieve a resting heart rate of not more than 70 beats/minute, she was given oral metoprolol $50 \mathrm{mg}$, and lorazepam 1mg. In addition, sublingual glyceryltrinitrate $0.5 \mathrm{mg}$ was given immediately before the scan to improve visualization of the coronary artery. Electrocardiographically (ECG)-gated CT coronary angiogram was performed by intravenous injection of $50 \mathrm{ml}$ of non-ionic low osmolar contrast medium (Iopromide 370 $\mathrm{mg} / \mathrm{mL}$ ) via 18 gaugebranula at a rate of $5 \mathrm{~mL} / \mathrm{s}$. It was followed by $50 \mathrm{ml}$ of $0.9 \%$ normal saline.

The CT scan revealed a tortuous fistulous communication between the left circumflex artery and the coronary sinus $13.6 \mathrm{~mm}$ in diameter for the left circumflex artery and $11.8 \mathrm{~mm}$ (axial) and $24 \mathrm{~mm}$ (coronal) diameter for the coronary sinus (Figures 2 and 3 ). The circumflex artery was remarkably large and originated directly from the left coronary cusp of the aorta. Left anterior descending artery originated from the left circumflex artery. The coronary sinus was bulky in size draining into the right

Copyright (C) 2015, Tehran University of Medical Sciences and Iranian Society of Radiology. This is an open-access article distributed under the terms of the Creative Commons Attribution-NonCommercial 4.0 International License (http://creativecommons.org/licenses/by-nc/4.0/) which permits copy and redistribute the material just in noncommercial usages, provided the original work is properly cited. 
atrium causing right atrial enlargement due to volume overload. A final diagnosis of left circumflex to coronary sinus fistula was made.

The patient was referred for cardiac catheterization, which confirmed a giant coronary arterial-venous (A-V) fistula arising from a dilated and mildly tortuous circumflex artery (Figure 4 ). The vessel course could not be well delineated because of its poor contrast opacification. At present, the patient is still waiting to undergo surgical closure of the fistula.
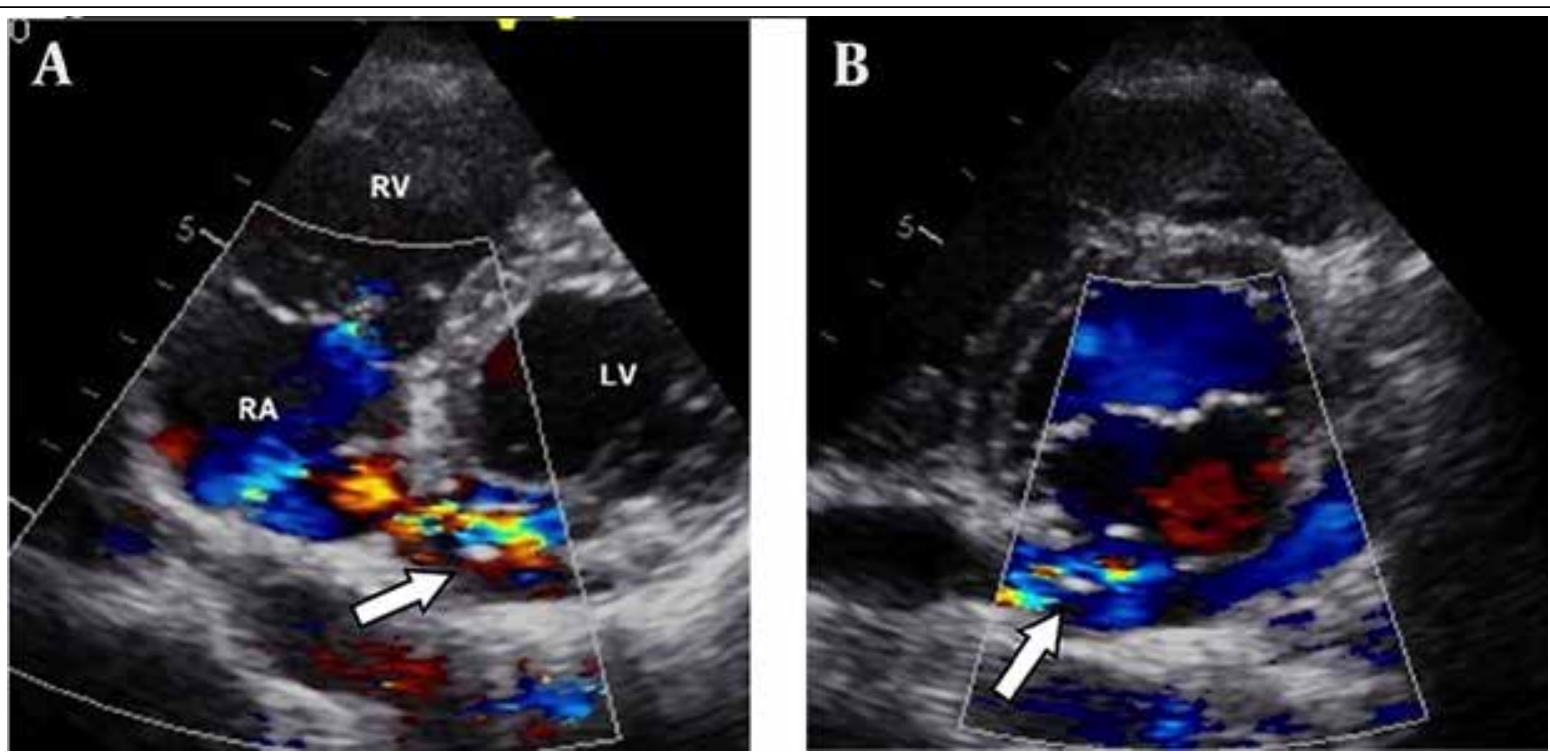

Figure 1. A 35-year-old woman with recurrent attacks of palpitation since childhood. A, Transthoracic color Doppler echocardiography shows the dilated left circumflex artery (arrow) emptying into the big coronary sinus. B, Continuous turbulence flow on color Doppler at the level of coronary arterycoronary sinus connection as well as in the coronary sinus indicating a left-to-right shunts flow.
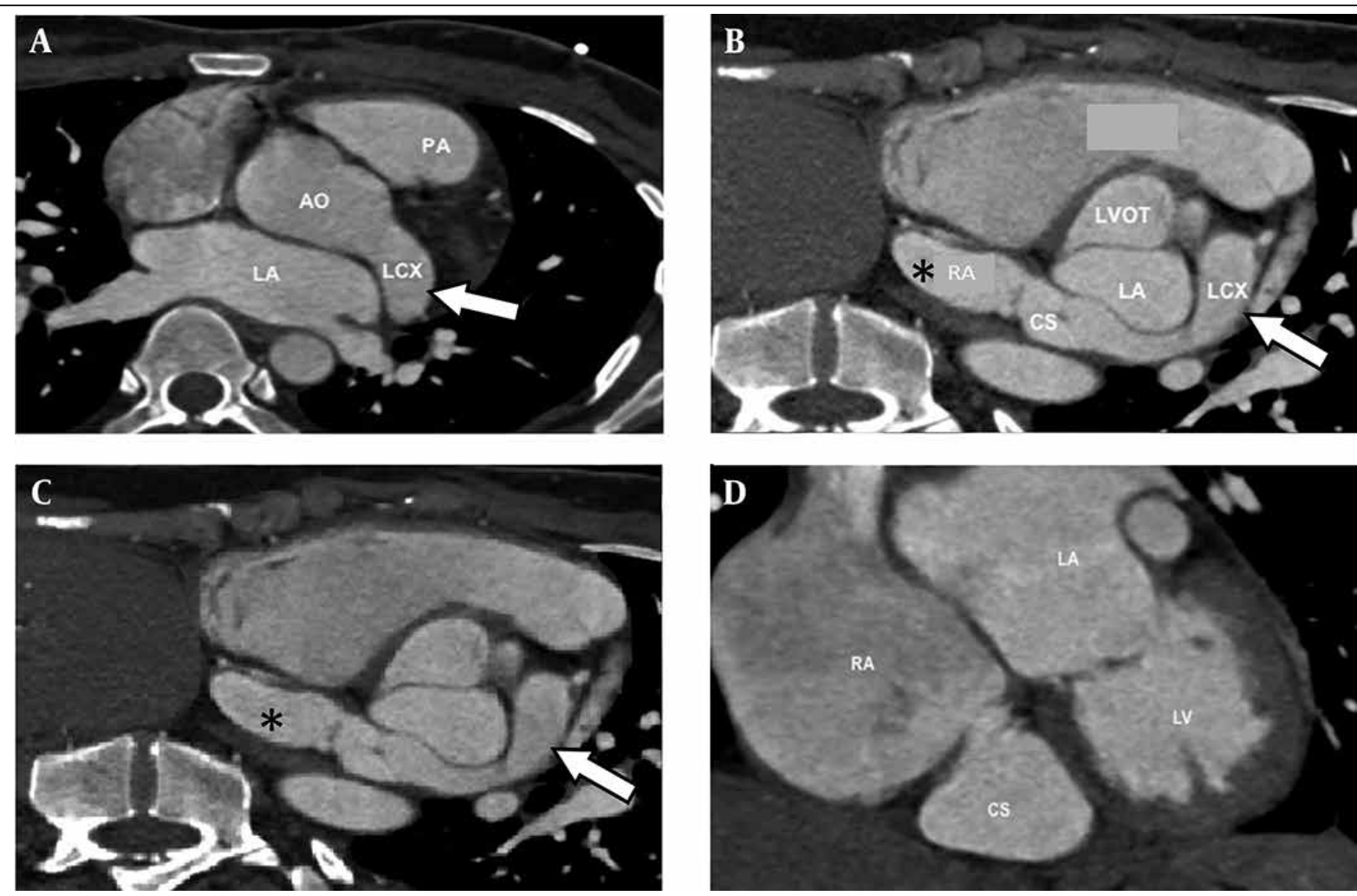

Figure 2. Axial (A) and reconstructed images (B and C) of coronary CT shows the dilated left circumflex artery (arrow) arising from the left coronary cusp and drains into the right atrium $\left({ }^{*}\right) . \mathrm{AO}=$ Aorta, $\mathrm{CS}=$ Coronary sinus, $\mathrm{LA}=$ Left atrium, LCX = Left circumflex artery, $\mathrm{LV}=$ Left ventricle, $\mathrm{LVOT}=\mathrm{Left}$ ventricular outflow tract, $\mathrm{PA}=$ Pulmonary artery, RA = Right atrium 

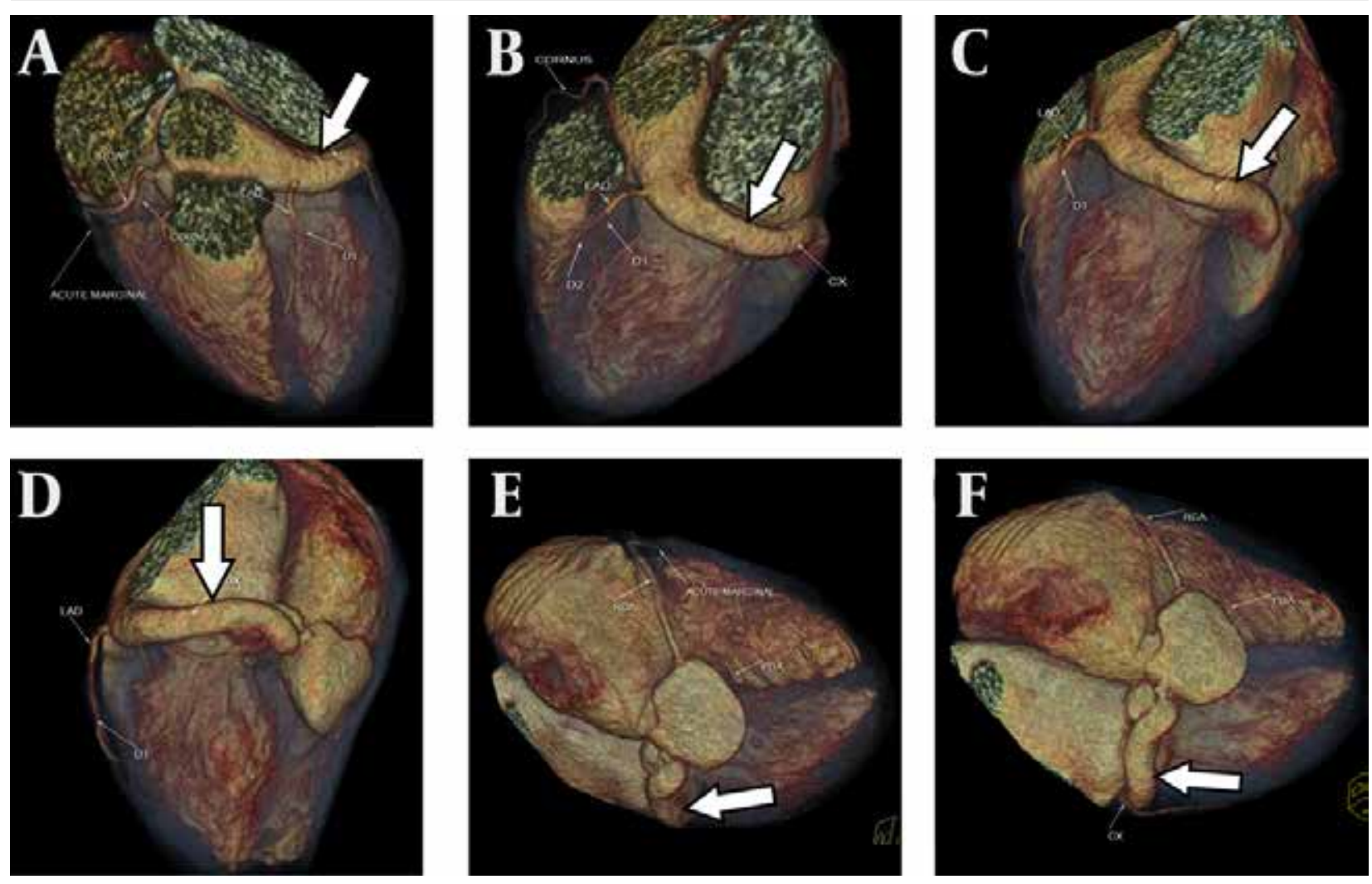

Figure 3. A-F. Volume rendering images of coronary CT show dilated left circumflex artery (Cx) (arrow).

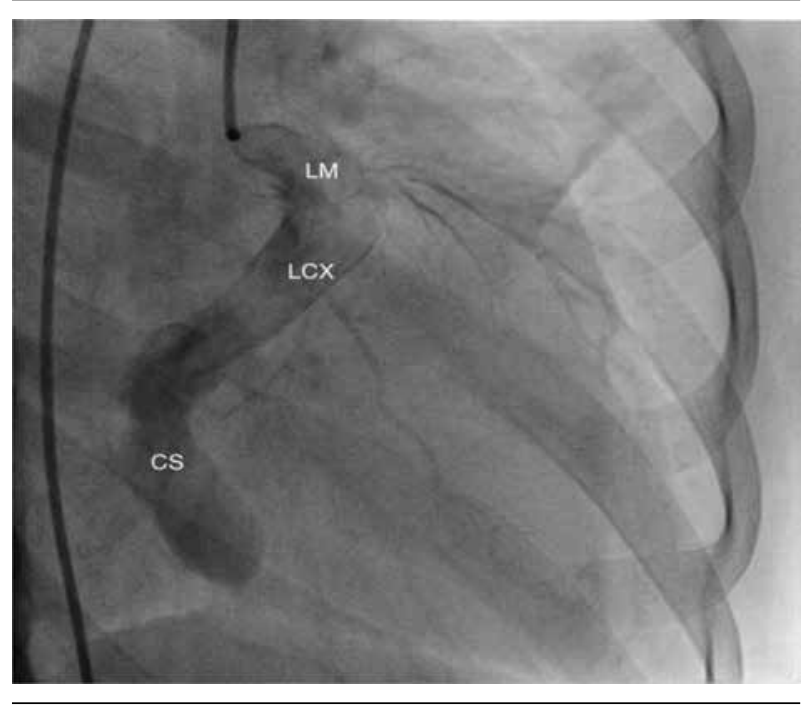

Figure 4. Coronary angiography (anteroposterior view) demonstrates contrast opacified left circumflex artery that is markedly dilated. CS $=$ Coronary sinus, LCX = Left circumflex artery, LM = Left main coronary artery

\section{Discussion}

Coronary artery fistulas (CAF) are anomalous terminations of the coronary arteries and are considered a major congenital anomaly (4). CAF is a rare abnormality and usually occurs alone (5). Congenital CAFs are thought to arise as a result of incomplete embryonic development; normally the coronary arteries communicate with the great vessels and chambers of the heart via sinusoids and during development, these sinusoids transform into a normally calibrated capillary network. It has been postulated that incomplete closure of these sinusoids can result in CAF (4). Acquired CAF can occur as a result of inflammation, atherosclerosis, trauma and collagen vascular disease. In our case, the patient had no significant previous surgical or medical history to suggest that the CAF is acquired. The cause of CAF in our patient is most likely congenital. The main difference between congenital and acquired CAF is mainly based on history. In terms of vessels involvement and symptoms, the congenital and acquired forms can appear identical (6).

CAF originates from the right coronary artery in $52 \%$ of cases followed by left anterior descending artery in 30\% and left circumflex in $18 \%$ of cases (5). More than $90 \%$ of CAFs drain into the right side of the heart (5). The most common drainage sites in order of decreasing frequency are the right ventricle (41\%), right atrium (26\%), pulmonary artery (17\%), coronary sinus (7\%), left atrium (5\%), left ventricle $(3 \%)$ and the superior vena cava $(1 \%)(4,7)$. Most CAFs are single communication, but multiple fistulae have been reported too (8).

The clinical symptoms of CAF depend on their anatomy, the relative size of the fistula and their flow reserve ( 9 , 10). The majority of patients with CAF are asymptomatic 
especially during the first two decades of life $(5,11,12)$. The most common clinical finding in this group of patients is continuous heart murmur that leads to further evaluation and diagnosis of CAF (8). This murmur is usually heard at the middle left or right sternal border or even at the lower sternal border (9). However, symptoms can develop later in life due to gradual enlargement of the fistula leading to an increase in the left to right shunt (8). At this stage, the patient can present with symptoms of congestive heart failure that presents as reduced effort tolerance and paroxysmal nocturnal dyspnea. Apart from that, they can also present with myocardial ischemic symptoms such as angina due to coronary artery steal $(5,8,11)$. Rare complications include stroke, endocarditis, endarteritis, fistula thrombosis that may cause acute myocardial infarction, atrial and ventricular arrhythmia $(5,12)$. In this case, our patient presented with palpitation and blackout that are rare presentations of CAF, but may happen as a result of atrial or ventricular arrhythmia. Otherwise, she did not show any findings of heart failure or angina.

There are various imaging modalities available for coronary artery evaluation. Tortuous and ectatic coronary artery is an important clue of CAF; however, the tortuous vascular course also makes the detailed anatomy difficult with echocardiogram.

Cardiac catheterization and angiography has traditionally been the main diagnostic technique in the assessment of CAF. It provides detailed anatomy of the fistula (5). Nevertheless, this technique only provides 2-dimensional depiction of a 3-dimensional structure. The precise course of the coronary artery and its relation to the surrounding structure is not shown. In addition, this modality is invasive with a small but not negligible procedurerelated morbidity and mortality.

Most of CAFs are small and are found incidentally during coronary angiography. However, with more frequent use of multidetector computed tomography (MDCT) in cardiac imaging, the number of incidental findings of CAF has been increasing. In contrast to catheter angiography, CT coronary angiography is noninvasive and has multiplanar capability to evaluate CAF. With the introduction of MDCT and the development of ECG-gated scanning and reconstruction technique, CT coronary angiography has emerged as a new opportunity for noninvasive cardiac imaging $(13,14)$. The high spatial and temporal resolution of MDCT with faster volume coverage permits direct visualization and analysis of the coronary artery system to detect any enlarged fistula (13). It allows systematic evaluation of the aorta, pulmonary vessels, cardiac chamber and ventriculoatrial connection as well as evaluation of aneurysmal dilatation or thrombus formation. In addition, CT via volume rendering enables 3-dimensional CT data evaluation of the heart and coronary arteries and gives an excellent overview of the complex cardiovascular anatomy for pre-operative planning (14). Furthermore, multiplanar reconstruction (MPR) analy- sis permits specification of the drainage site in a patient with CAF $(13,14)$. In congenital CAF, the involved coronary artery is usually tortuous and dilated, as in this case. True aneurysm formation can also be seen along the fistulous tract in the congenital form.

Magnetic resonance imaging (MRI) is another noninvasive technique that can be used in the assessment of CAF to evaluate anatomy, flow and function. Cine MRI sequence has the advantage of demonstrating dynamics and turbulence flow at the fistula entry site (9). Black blood imaging allows better visualization of the coronary lumen and wall over conventional spin echo sequence by improving the image quality (9). Besides that, MRI has the superiority of not using ionizing radiation. However, it is not as widely available as CT and requires longer scanning time.

Spontaneous closure of the fistula is uncommon and may occur due to spontaneous thrombosis especially in small CAFs (4). Most CAFs will eventually enlarge and warrant intervention by either trans catheter closure or surgical ligation. Indication of treatment includes symptoms attributable to CAF, multiple fistula connection, myocardial ischemia and fistula rupture with cardiac tamponade $(11,15)$. Trans catheter closure is usually indicated for proximal location of the fistulous vessel, single drain site, extra anatomic termination of fistula away from the normal coronary artery and older age group (10). On the other hand, surgical ligation is indicated for large CAFs, multiple communications, multiple terminations, very tortuous pathway, significant aneurysm formation and presence of large vascular branches (10). Trans catheter occlusion is associated with lower morbidity and mortality rate as compared with surgical ligation (15). Long-term follow-up is recommended due to possible post-operative recanalization, persistent dilatation of the involved artery, thrombus formation and myocardial infarction (16).

Management of CAF (either congenital or acquired) mainly depends on the symptoms and size of the fistula. Small CAF in an asymptomatic patient does not require immediate intervention but a regular follow up and reassessment of the fistula are indicated (15). However, asymptomatic CAF should be repaired as symptoms and fistula related complications increase with age $(8,12)$. Furthermore, postoperative morbidity and mortality is higher among the older age group. For this reason, our patient was planned for surgical ligation.

In conclusion, even though cardiac catheterization and angiogram is the gold standard diagnostic method, MDCT is a noninvasive and useful alternative in determining the accurate anatomic relationship in CAF. Therefore, in addition to evaluation of the coronary arteries for stenosis and plaques, special attention should be paid to their courses and terminations in every CT study of the heart to detect these potentially fatal anomalies. The choice between embolization and surgical ligation should depend on anatomical and functional characteristics of the fistula. 


\section{References}

1. Krause W. [Uber den ursprung einer accessorischen a Coronaria aus der a.]. Z Ratl Med Pulmonalis. 1865;24:225-7.

2. Wenger NK. Rare causes of coronary artery disease. JW Hurst editor New York: McGraw-Hill; 1978.

3. Lin FC, Chang HJ, Wen MS, Yeh SJ, Wu D. Multiplane transesophageal echocardiography in the diagnosis of congenital coronary artery fistula. Am Heart J. 1995;130(6):1236-42.

4. Hirooka K, Hanatani A, Nakatani S, Yasumura Y, Bando K, Miyatake $\mathrm{K}$, et al. Huge saccular aneurysm in a coronary-pulmonary fistula fed by the left and right coronary arteries. Circ $J$. 2002;66(5):525-7.

5. Qureshi SA. Coronary arterial fistulas. Orphanet $J$ Rare Dis. 2006;: :51

6. Said SA, el Gamal MI. Coronary angiographic morphology of congenital coronary arteriovenous fistulas in adults: report of four new cases and review of angiograms of fifteen reported cases. Cathet Cardiovasc Diagn. 1995;35(1):29-35.

7. Lin FC, Chang HJ, Chern MS, Wen MS, Yeh SJ, Wu D. Multiplane transesophageal echocardiography in the diagnosis of congenital coronary artery fistula. Am Heart J. 1995;130(6):1236-44.

8. Ata Y, Turk T, Bicer M, Yalcin M, Ata F, Yavuz S. Coronary arteriovenous fistulas in the adults: natural history and management strategies. JCardiothorac Surg. 2009;4:62.
9. Parga JR, Ikari NM, Bustamante LN, Rochitte CE, de Avila LF, Oliveira SA. Case report: MRI evaluation of congenital coronary artery fistulae. Br J Radiol. 2004;77(918):508-11.

10. Cebi N, Schulze-Waltrup N, Fromke J, Scheffold T, Heuer H. Congenital coronary artery fistulas in adults: concomitant pathologies and treatment. Int J Cardiovasc Imaging. 2008;24(4):349-55.

11. Yilmaz R, Demirbag R, Gur M. Echocardiographic diagnosis of a right coronary artery-coronary sinus fistula. Int J Cardiovasc Imaging. 2005;21(6):649-54.

12. Liberthson RR, Sagar K, Berkoben JP, Weintraub RM, Levine FH Congenital coronary arteriovenous fistula. Report of 13 patients, review of the literature and delineation of management. Circulation. 1979;59(5):849-54.

13. Sato Y, Mitsui M, Takahashi H, Miyazawa T, Okabe H, Inoue F, et al. A giant left circumflex coronary artery-right atrium arteriovenous fistula detected by multislice spiral computed tomography. Heart Vessels. 2004;19(1):55-6.

14. Seon HJ, Kim YH, Choi S, Kim KH. Complex coronary artery fistulas in adults: evaluation with multidetector computed tomography. Int J Cardiovasc Imaging. 2010;26(Suppl 2):261-71.

15. Stougiannos PN, Danias PG, Karatzis EN, Kakkavas AT, Trikas AG. Incidental diagnosis of a large coronary fistula: angiographic and cardiac MRI findings. Hellenic J Cardiol. 2011;52(1):75-8.

16. Gowda RM, Vasavada BC, Khan IA. Coronary artery fistulas: clinical and therapeutic considerations. Int J Cardiol. 2006;107(1):7-10. 